\title{
Application of Creatine Kinase Isoenzymes for Detecting Pathophysiological Changes in Yellowtail Infected with Lactococcus garvieae
}

\author{
Hae-Kyung Lee ${ }^{1}$, Masashi Maita ${ }^{1 *}$, Yutaka Fukuda ${ }^{2}$ \\ and Nobuaki Okamoto ${ }^{1}$ \\ ${ }^{1}$ Department of Aquatic Biosciences, Tokyo University of Fisheries, \\ Konan 4, Minato, Tokyo 108-8477, Japan \\ ${ }^{2}$ Oita Institute of Marine and Fisheries Science, Kamiura, \\ Oita 879-2601, Japan
}

(Received January 28, 1999)

\begin{abstract}
Creatine kinase (CK) isoenzymes were examined as diagnostic indicators for pathophysiological changes in yellowtail infected with Lactococcus garvieae. Three isoenzymes of CK similar to those found in human were detected by agarose electrophoresis. CK isoenzymes in the brain, heart and intestine consisted of CK-MM, CK-MB and CK-BB. CK-BB was not detected in other tissues or plasma. Normal yellowtail plasma contained CK-MM and CK-MB, while CK-BB and CK-SP appeared in the plasma of infected fish. This phenomenon was found in both naturally and artificially infected fish. These results suggest that appearance of CK-BB and CK-SP in the plasma may reflect the pathophysiological changes due to the infection. Therefore, we consider that the examination of plasma CK isoenzymes is a pathophysiological diagnostic tool for the streptococcal infection in yellowtail.
\end{abstract}

Key words: creatine kinase, Lactococcus garvieae, isoenzyme, yellowtail, diagnosis, Seriola quinqueradiata, pathophysiology

Lactococcus garvieae infection has caused considerable economic losses in cultured yellowtail in Japan. Though bacteriological examination is essential for diagnosis of the disease, observation of pathophysiological changes in infected fish helps indirect diagnosis. We consider that pathophysiological diagnosis for bacterial infection in fish offer useful information concerning the severity of the disease, and culturists may be able to adopt suitable measures for preventing the disease based on such information. Blood examination is one of the means to know the changes. While it has been found that plasma alkaline phosphatase activity and glucose level increased in fish infected with Lactococcus garvieae or Streptococcus iniae (Ikeda and Minami, 1982; Kusuda and Ninomiya, 1992), it is still unknown which tissue injury relates to those changes. As typical symptoms of this disease are encephalitis and pericarditis, we consider that some injuries of brain and heart due

\footnotetext{
* Corresponding author

E-mail: mmaita@ tokyo-u-fish.ac.jp
}

to the disease may give diagnostic indicators for $L$. garvieae infection.

Changes in creatine kinase (CK; E.C. 2.7.3.2) reflect pathological alterations in specific human organs. $\mathrm{CK}$ is a dimer of two subunits of, $M$ and $B$, generating three different isoenzymes in mammalian cells: MM (CK-MM, muscle type), BB (CK-BB, brain type) and MB (CK-MB, myocardial type). In human, distribution of $C K$ isoenzymes is tissue specific, e.g. skeletal muscle contains CK-MM, while brain and heart contains CK-BB and CK$M B$, respectively. Thus, $C K$ isoenzymes are frequently used as serum diagnostic markers for acute myocardial infraction, skeletal muscle disease and neurological injury (Sirag et al., 1986; Hamburg et al., 1991). If CK isoenzymes are also tissue specific in yellowtail like in human, examination of plasma CK isoenzymes in yellowtail may detect brain and heart injuries due to the disease.

Enzymological properties of $\mathrm{CK}$ in red sea bream, pacific mackerel and carp were studied by Nakagawa and Nagayama (1991). Bucher (1990) investigated the distribution of CK activity in organs of rainbow trout. 
Variation of CK isoenzymes has been studied in skeletal muscle, heart, brain and stomach of salmonid fish (Perriard et al., 1972; Ferris and Whitt, 1978; White et al., 1992). Williams et al. (1996) reported plasma CK isoenzyme patterns of healthy rainbow trout. It is known that the severe myopathy in "Sudden death syndrome" of Atlantic salmon was accompanied by a significant elevation of CK activity levels (Rodger et al., 1991). The present paper describes distribution of CK isoenzymes in organs of yellowtail and verify whether plasma CK isoenzymes can be used for the diagnosis of the $L$. garvieae infection in yellowtail.

\section{Material and Methods}

Sample preparation, measurement of CK activity and analysis of CK isoenzymes for tissue distribution

Six healthy yellowtail Seriola quinqueradiata weighing 80-120 g were obtained from Oita Institute of Marine and Fisheries Science, Oita Prefecture, Japan. Blood samples were taken by cardiac puncture with heparinized syringes. The plasma was separated by centrifuging at $1,000 \times g, 10 \mathrm{~min}$ at $4^{\circ} \mathrm{C}$ and stored at $-80^{\circ} \mathrm{C}$ until used. Pieces of white muscle, brain, heart, intestine, kidney, spleen and liver taken from each fish were washed three times in Ringer's solution and stored at $-80^{\circ} \mathrm{C}$. The tissue materials were homogenized with $0.25 \mathrm{M}$ sucrose solution on ice. The homogenates were centrifuged at $10,000 \mathrm{rpm}$ for $30 \mathrm{~min}$ at $4^{\circ} \mathrm{C}$ and the supernatants were used for the analysis of CK activity and the isoenzymes.

CK activity was assayed using commercial kit (Oliver's method, Wako). Protein concentration of the analytical samples, except the plasma, was measured according to Lowry's method (1951). CK activities of each tissue were expressed as $\mathrm{mU} / \mathrm{mg}$ protein.

Agarose gel electrophoresis was carried out by the clinical laboratory procedure using precast "special agarose electrophoresis film" with commercial electrophoresis system (CIBA Corning Co.). Samples were applied to the gel when the CK activity reached 1,000 $\mathrm{mU} / \mathrm{mL}$. Standard human serum was used for checking the separation of $\mathrm{CK}$ isoenzymes.

\section{Examination of CK isoenzymes in yellowtail infected with L. garvieae}

Plasma samples were collected from fish naturally infected with L. garvieae, occuring in a farm in Kagoshima Prefecture. Bacteria isolated from the brain and/or pericardia of twenty one samples were examined for L. garvieae.

Artificial infection was done to confirm the results obtained from naturally infected fish. Ninety non infected fish weighing $110 \mathrm{~g}$ on the average were placed in $2 \mathrm{~m}^{3}$ tanks for challenge test and adjusted for 1 week. Lactococcus garvieae strain OT62262 isolated from diseased yellowtail in Oita Prefecture in 1996 was

employed in the experiment. The bacterium was cultured in Tood Hewitt Broth (Nissui) at $25^{\circ} \mathrm{C}$ for $24 \mathrm{hr}$ and used. Fish were challenged by immersion in seawater containing L. garvieae OT62262 cells at a concentration of $4.1 \times 10^{8} \mathrm{CFU} / \mathrm{mL}$ for $15 \mathrm{~min}$. Following the challenge, fish were held at water temperature of $23.0-25.0^{\circ} \mathrm{C}$. Five fish each were caught by spoon net at $0,1,2,3,4,5,8$ and 16 days post infection. Blood samples were obtained by the cardiac puncture using heparinized syringes fitted with $23 \mathrm{G}$ needles. Samples of the brain, heart, kidney and liver were dissected asceptically, washed with sterilized Ringer's solution several times, and homogenized. Viable numbers of L. garvieae were estimated from spread plates of serial dilutions on brain heart infusion agar (BHIA; Difco). The blood samples were centrifuged $(1,000 \times g, 10 \mathrm{~min})$ to separate the plasma, which was kept at $-80^{\circ} \mathrm{C}$ and later used for analysis. Plasma alkaline phosphatase activity was determined as reference using a CL-7100 automated biochemical analyzer (Shimadzu Co. Ltd.).

\section{Results}

Tissue distribution of CK activity and CK isoenzymes in yellowtail

Creatine kinase activity was detected in plasma and

\section{1}

2

3

4

5

6

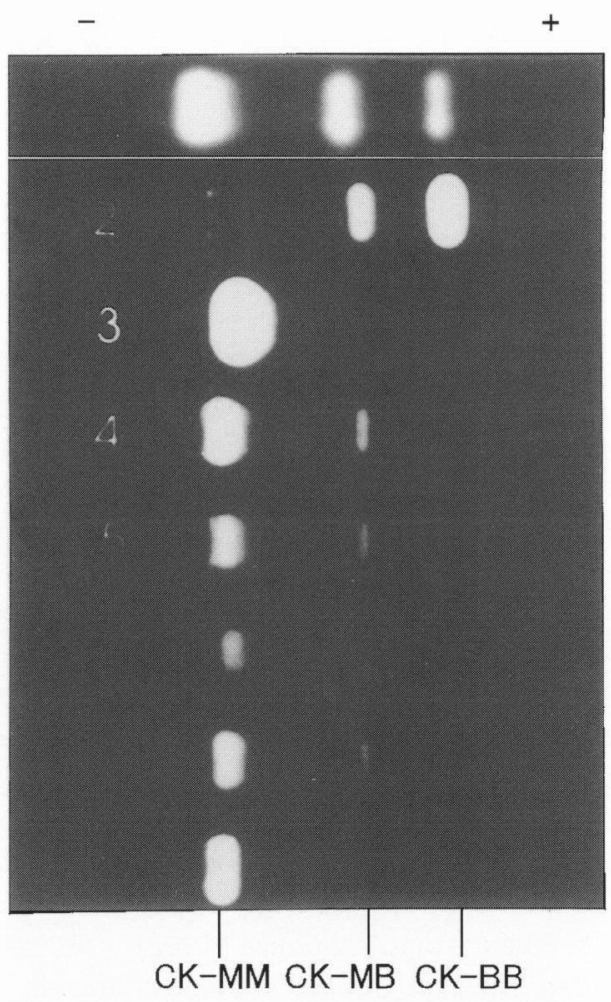

Fig. 1. Creatine kinase isoenzymes in normal yellowtail. Lane numbers are shown as follows; 1: Human standard, 2: Brain, 3: White muscle, 4: Liver, 5: Heart, 6: Spleen, 7: Kidney, 8: Plasma. CK-MM, CK-MB and CK-BB were referenced by the electrophoretic mobility of human standard. 

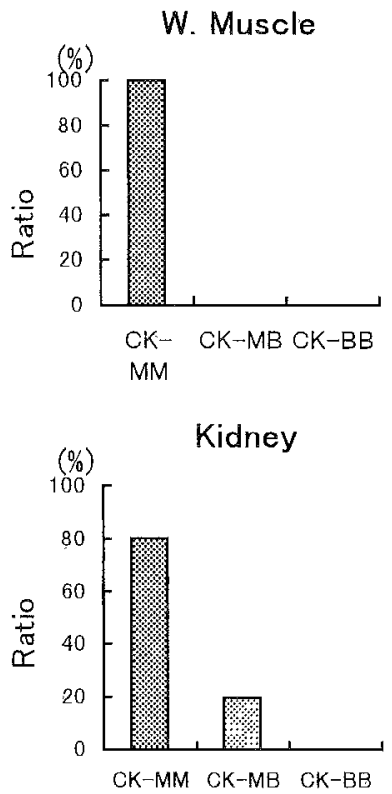
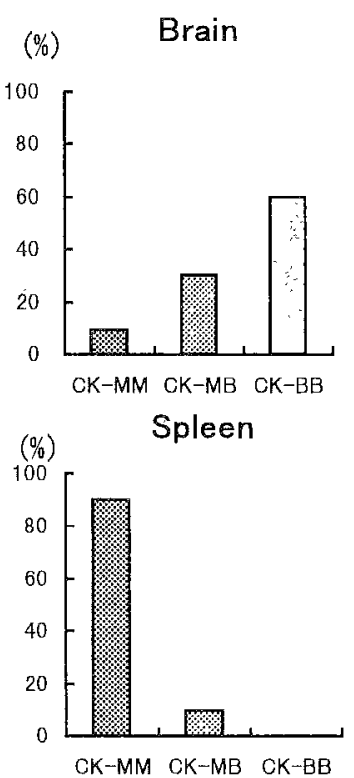

Heart
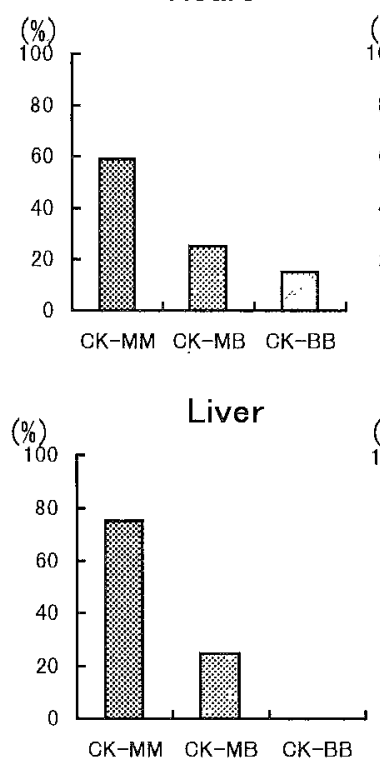

Intestine
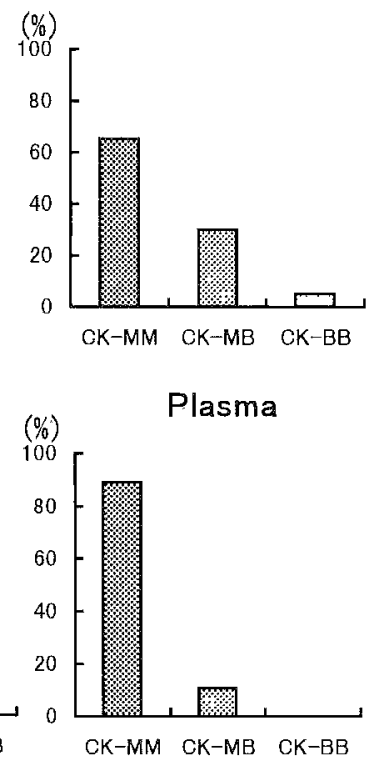

Fig. 2. Distribution of CK isoenzymes in tissues of normal yellowtail.

Ratios (\%) between CK isoenzymes in the tissues of were almost same in 6 fish examined. Therefore, a representative result is shown in this figure.

all tissues examined. The activity was $4,761 \pm 2,253$ in white muscle, $740 \pm 166$ in brain, $297 \pm 280$ in heart, $443 \pm 139$ in intestine, $148 \pm 45$ in kidney, $191 \pm 58$ in spleen, $87 \pm 116 \mathrm{mU} / \mathrm{mg}$ protein in liver and $41 \pm 26 \mathrm{mU} /$ $\mathrm{mL}$ in plasma. CK activity of white muscle was approximately 6-fold higher than that of the brain and more than 10-fold higher than those of other tissues. These results were similar to those obtained from rainbow trout (Bucher, 1990).

Three bands of CK isoenzymes appeared on agarose electrophoresis films, showing a similar pattern to human control (Fig. 1). CK-MM, CK-MB and CK-BB were tentatively identified by their electrophoretic mobility compared with the human standard serum. CK-MM could only be found in the white muscle, while CK-MM and $\mathrm{CK}-\mathrm{MB}$ were observed in the kidney, spleen and liver. CK isoenzymes of the brain, heart and intestine consisted of three isoenzymes (CK-MM, CK-MB and CK-BB). CK-MM and CK-MB were detected in the healthy yellowtail plasma, while CK-BB could not be detected (Fig. 2).

\section{Plasma CK isoenzymes in fish naturally infected with L. garvieae}

Electrophoretic pattern of plasma CK isoenzymes in fish naturally infected with L. garvieae is shown in Fig. 3. While uninfected fish showed CK-MM and/or both CK-MM and CK-MB, diseased fish exhibited CK-BB and a novel band (CK-SP) in addition to CK-MM and CK-MB. CK-BB was detected in 12 among 21 diseased fish $(57 \%)$. On the other hand, CK-SP were observed in 17 of infected fish (81\%). Four diseased fish (19\%) neither had CK-BB nor CK-SP like the healthy fish.

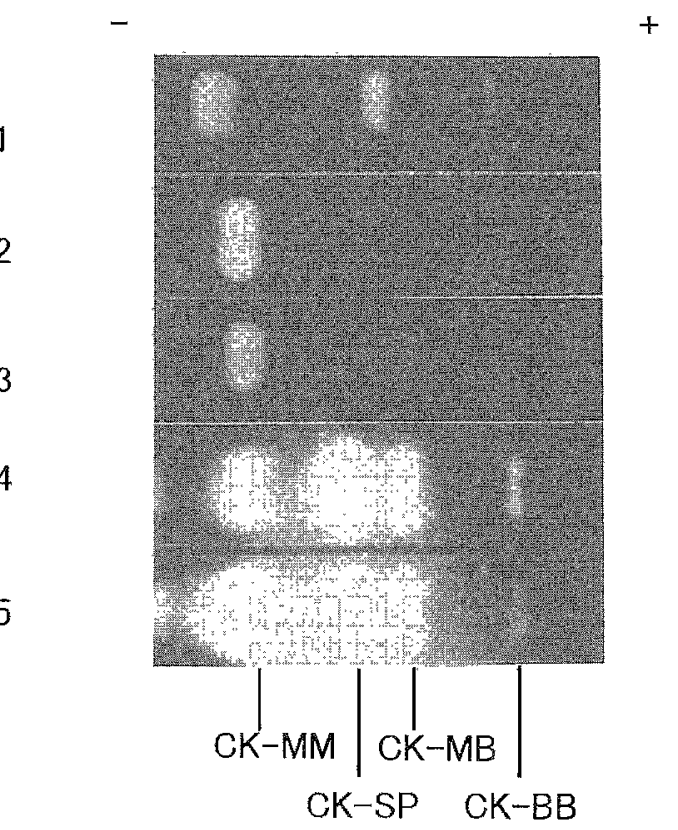

Fig. 3. Plasma CK isoenzymes in yellowtail naturally infected with L. garvieae.

Lane numbers are shown as follows; 1 : Human standard, 2 and 3: Healthy fish, 4 and 5: Infected fish. A novel band (CK-SP) is found at the position between CK-MM and CK-MB.

Plasma CK isoenzymes in fish artificially infected with $L$. garvieae

Individual data of experimental fish are shown in Table 1. In artificial infection, L. garvieae was isolated from some organs (at least from one organ) in all examined fish from 1 to 8 days post infection. CK-BB was detected only in 1 among 5 fish and CK-SP was not 
Table 1. Individual data of CK isoenzymes, plasma alkaline phosphatase activity and log numbers of Lactococcus garvieae in artifical infection

\begin{tabular}{|c|c|c|c|c|c|c|c|c|}
\hline \multirow{2}{*}{ Specimen } & \multirow{2}{*}{ No. } & \multicolumn{2}{|c|}{ CK isoenzymes* } & \multirow{2}{*}{$\begin{array}{c}\text { Alkaline } \\
\text { phosphatase } \\
\text { (IU/L) }\end{array}$} & \multicolumn{4}{|c|}{ Log number of $L$. garvieae } \\
\hline & & CK-BB & CK-SP & & Brain & Heart & Kidney & Liver \\
\hline \multirow{5}{*}{ Pre-infection } & 1 & ND & ND & 153 & ND & ND & ND & ND \\
\hline & 2 & ND & ND & 198 & ND & ND & ND & ND \\
\hline & 3 & ND & ND & 174 & ND & ND & ND & ND \\
\hline & 4 & ND & ND & 200 & ND & ND & ND & ND \\
\hline & 5 & ND & ND & 210 & ND & ND & ND & ND \\
\hline \multirow{5}{*}{$\begin{array}{c}1 \text { day } \\
\text { post infection }\end{array}$} & 1 & ND & ND & 138 & ND & 3.40 & ND & ND \\
\hline & 2 & $\mathrm{D}$ & ND & 115 & ND & ND & 1.95 & ND \\
\hline & 3 & ND & ND & 177 & ND & ND & 4.84 & 1.48 \\
\hline & 4 & ND & ND & 135 & 2.46 & 3.95 & 3.68 & 3.23 \\
\hline & 5 & ND & ND & 166 & ND & ND & 3.18 & 3.04 \\
\hline \multirow{5}{*}{$\begin{array}{c}2 \text { day } \\
\text { post infection }\end{array}$} & 1 & ND & D & 289 & 5.26 & 5.00 & 5.79 & 4.90 \\
\hline & 2 & ND & $D$ & 540 & 4.95 & 3.30 & 6.00 & 6.34 \\
\hline & 3 & ND & $D$ & 634 & 6.32 & 5.81 & 5.70 & 5.69 \\
\hline & 4 & $D$ & ND & 328 & 5.90 & 4.87 & 5.70 & 6.15 \\
\hline & 5 & ND & ND & 688 & 7.93 & 4.91 & 5.88 & 6.34 \\
\hline \multirow{5}{*}{$\begin{array}{c}3 \text { day } \\
\text { post infection }\end{array}$} & 1 & ND & D & 1,032 & 6.45 & 3.30 & 4.15 & 3.34 \\
\hline & 2 & ND & $D$ & 1,338 & 6.94 & 5.80 & 5.78 & 4.67 \\
\hline & 3 & ND & $D$ & 802 & 8.11 & 6.51 & 5.52 & 5.30 \\
\hline & 4 & ND & $D$ & 76 & 5.69 & 4.79 & 1.60 & 4.79 \\
\hline & 5 & ND & D & 2,480 & 5.70 & 7.40 & 4.60 & 3.59 \\
\hline \multirow{5}{*}{$\begin{array}{c}4 \text { day } \\
\text { post infection }\end{array}$} & 1 & D & D & 783 & 6.77 & 5.90 & 5.64 & 4.11 \\
\hline & 2 & ND & $D$ & 550 & 2.20 & 6.40 & 3.11 & ND \\
\hline & 3 & $D$ & ND & 73 & ND & 3.26 & ND & ND \\
\hline & 4 & $D$ & $D$ & 760 & 2.78 & 5.90 & 3.77 & 3.92 \\
\hline & 5 & D & D & 685 & 6.89 & 5.15 & 5.94 & 5.38 \\
\hline \multirow{5}{*}{$\begin{array}{c}5 \text { day } \\
\text { post infection }\end{array}$} & 1 & ND & D & 1,716 & 8.28 & 6.88 & 6.30 & 5.00 \\
\hline & 2 & $D$ & D & 1,632 & 6.69 & 7.60 & 6.00 & 5.08 \\
\hline & 3 & ND & $D$ & 507 & 4.52 & 7.49 & 4.81 & 5.69 \\
\hline & 4 & ND & $D$ & 984 & 7.72 & 5.72 & 5.41 & 4.11 \\
\hline & 5 & D & D & 1,255 & 7.80 & 8.00 & 5.68 & 5.66 \\
\hline \multirow{5}{*}{$\begin{array}{c}8 \text { day } \\
\text { post infection }\end{array}$} & 1 & ND & ND & 78 & 6.86 & 6.49 & 6.18 & 4.70 \\
\hline & 2 & $D$ & D & 1,325 & 6.83 & 7.08 & 5.70 & 4.85 \\
\hline & 3 & $D$ & ND & 188 & 6.90 & 6.91 & 5.20 & 5.46 \\
\hline & 4 & D & $D$ & 937 & 7.94 & 6.08 & 4.57 & 5.28 \\
\hline & 5 & ND & D & 623 & 7.48 & 7.20 & 3.90 & 3.95 \\
\hline \multirow{5}{*}{$\begin{array}{c}16 \text { day } \\
\text { post infection }\end{array}$} & 1 & D & ND & 183 & ND & ND & ND & ND \\
\hline & 2 & $D$ & ND & 172 & ND & ND & ND & ND \\
\hline & 3 & $D$ & ND & 142 & ND & ND & ND & ND \\
\hline & 4 & D & ND & 126 & ND & ND & ND & ND \\
\hline & 5 & $D$ & ND & 118 & ND & ND & ND & ND \\
\hline
\end{tabular}

* Results of creatine kinase (CK) isoenzymes present as follows; ND: not detected, D: detected.

detected in any fish at 1 day of post infection. Incidence of fish with CK-SP $(80 \%)$ was higher than those of fish with CK-BB $(40 \%)$ during the period from 2 to 8 days post infection. CK-SP was detected in almost all fish in which alkaline phosphatase activity was higher than the initial value. Such positive correlation was not observed between CK-BB and alkaline phosphotase. Incidence of fish in which neither CK-BB nor CK-SP could be detected was $8 \%$ of all the fish infected with the bacteria. Although the bacteria were not reisolated, CK-BB was observed in fish examined at 16 days post infection.

\section{Discussion}

Examination of plasma CK isoenzymes is useful to know the pathophysiological changes in yellowtail infected with Lactococcus garvieae. Our results indicate that CK isoenzymes of yellowtail are tissue specific and the plasma normally contains two CK isoenzymes, CK$\mathrm{MM}$ and $\mathrm{CK}-\mathrm{MB}$. CK-BB and CK-SP, which are unusual in the plasma of healthy fish, were detected in that of fish infected with $L$. garvieae and the phenomenon was confirmed in both naturally and artificially infected fish. 
It is important to know which tissue produces CK-BB and/or CK-SP appeared in the plasma. Though not confirmed, CK-BB in the plasma of infected fish may reflect leakage from some of the affected brain, heart and intestine because CK-BB was found only in those tissues. On the other hand, CK-SP was not contained in any tissue of the healthy fish. It is not evident that CK-SP relates to the injury of tissues. However, we consider that the presence of CK-SP in the plasma of infected fish reflect some pathophysiological change due to the disease. Further investigations of CK-SP is needed to understand better the pathophysiological condition of the diseased fish.

Examination of CK isoenzymes has an advantage over measurement of plasma alkaline phosphatase activity and glucose level in pathophysiological diagnosis for $L$. garvieae infection. The occurrence of CK-SP was found to be related to high plasma alkaline phosphatase activity. It is well known that the alkaline phosphatase levels increase in fish infected with $L$. garvieae. The normal reference values of plasma components in yellowtail have not been established and variations of plasma components levels in individuals are wide. However, normal standard is not necessary for reference of $\mathrm{CK}$ isoenzymes because estimation of CK isoenzymes is carried out by detecting substances which healthy fish do not have. Hence it is considered that detection of $\mathrm{CK}$ enzymes is a more useful diagnostic tool to study pathophysiological changes in fish infected with L. garvieae. Plasma CK activity of rainbow trout is influenced by the effects of starvation, spawning and seasons (Bucher, 1990), but there is no report about physiological changes in the CK isoenzyme patterns in yellowtail. Thus, the influence of these factors on plasma CK isoenzymes has to be investigated in order to use them for diagnosis of L. garvieae infection in yellowtail.

\section{Acknowledgments}

We would like to thank Oita Institute of Marine and Fisheries Science for providing the fish used in this study and Mr. M. Fukudome (Kagoshima Prefectural Fisheries Experimental Station) for his kindly cooperation.

\section{References}

Bucher, F. (1990): Organ patterns and natural fluctuations of blood enzymes of rainbow trout (Salmo gairdneri rich.). Comp. Biochem. Physiol., 96B, 795-799.

Ferris, S. D. and G. S. Whitt (1978): Genetic and molecular analysis of nonrandom dimer assembly of the creatine kinase isozymes of fishes. Biochem. Genet., 16, 811-829.

Hamburg, R. J., D. L. Friedman and M. B. Perryman (1991): Metabolic and diagnostic significance of creatine kinase isoenzymes. Trend. Cardio. Med., 1, 195-200.

Ikeda, Y. and T. Minami (1982): Hematological and hemochemical assessment on streptococcal infection in cultured yellowtail. Bull. Japan. Soc. Sci. Fish., 48, 13831388.

Kusuda, R. and M. Ninomiya (1992): Measurement of hematological and hemochemical changes in yellowtail Seriola quinqueradiata, infected with Enterococcus seriolicida using of Vision system. Suisanzoshoku, 40, 323-328.

Lowry, O. H., N. J. Rosenbrough, A. L. Farr and R. J. Randall (1951): Protein measurement with Folin phenol regent. J. Biol. Chem., 193, 265-275.

Nakagawa, T. and F. Nagayama (1991): Enzyme properties of fish muscle creatine kinase. Comp. Biochem. Physiol., 98B, 349-354.

Perriard, J.-C., A. Scholl and H. M. Eppenberger (1972): Comparative studies on creatine kinase isozymes from skeletalmuscle and stomach of trout. J. Exp. Zool., 182, 119-126.

Rodger, H. D., T. M. Murphy., E.M. Drinan and D. A. Rice (1991): Acute skeletal myopathy in farmed Atlantic salmon Salmo salar. Dis. Aquat. Org., 12, 17-23.

Sirag, E. E., G. Gercken and K. Harm (1986): The isoelectric focusing of creatine kinase variants: II. The heterogeneity of creatine kinase in human serum with normal and elevated catalytic concentrations. J. Clin. Chem. Clin. Biochem., 24, 847-860.

White, K. C., P. C. Babbitt, D. D. Buechter and G. L. Kenyon (1992): The principal islet of the Coho salmon (Oncorhynchus kisutch) contains the BB isoenzyme of creatine kinase. J. Prot. Chem., 11, 489-494.

Williams, J., P. Stewart and P. Timpson (1996): Characterization of rainbow trout, Oncorhynchus mykiss (Walbaum), serum creatine kinase isoenzymes and isoforms by means of electrophoresis and isoelectric focusing. J. Fish Dis., 19, 441-447. 